\title{
Glucagon-Like Peptide-I Receptor Signaling in the Lateral Dorsal Tegmental Nucleus Regulates Energy Balance
}

\author{
David J Reiner', Rosa M Leon', Lauren E McGrath', Kieran Koch-Laskowski', Joel D Hahn², Scott E Kanoski ${ }^{3}$, \\ Elizabeth G Mietlicki-Baase ${ }^{1,4}$ and Matthew R Hayes*,I \\ 'Translational Neuroscience Program, Department of Psychiatry, Perelman School of Medicine, Department of Biological Sciences, University of \\ Pennsylvania, Center for Neurobiology and Behavior, Philadelphia, PA, USA; ${ }^{2}$ Neurobiology Section, University of Southern California, Los Angeles, \\ CA, USA; ${ }^{3}$ Human and Evolutionary Biology Section, University of Southern California, Los Angeles, CA, USA
}

\begin{abstract}
The neurobiological substrates that mediate the anorectic effects of both endogenous glucagon-like peptide-I (GLP-I) and exogenous GLP-I receptor (GLP-IR) agonists are an active area of investigation. As the lateral dorsal tegmental nucleus (LDTg) expresses the GLP-IR and represents a potential neuroanatomical hub connecting the nucleus tractus solitarius (NTS), the major central source of GLP-I, with the other nuclei in the midbrain and forebrain, we tested the hypothesis that GLP-IR signaling in the LDTg controls food intake. Direct activation of LDTg GLP-IR suppresses food intake through a reduction in average meal size and independent of nausea/malaise. Immunohistochemical data show that GLP-I-producing neurons in the NTS project to the LDTg, providing anatomical evidence of endogenous central GLP-I in the LDTg. Pharmacological blockade of LDTg GLP-IRs with exendin-(9-39) dose-dependently increases food intake and attenuates the hypophagic effects of gastric distension. As GLP-I mimetics are administered systemically in humans, we evaluated whether peripherally administered GLP-IR agonists access the LDTg to affect feeding. Immunohistochemical data show that a systemically administered fluorescent GLP-IR agonist accesses the LDTg and is juxtaposed with neurons. Additionally, blockade of LDTg GLP-IRs attenuates the hypophagic effects of a systemic GLP-IR agonist. Together, these data indicate that LDTg GLP-IR signaling controls energy balance and underscores the role of the LDTg in integrating energy balance-relevant signals to modulate feeding. Neuropsychopharmacology (2018) 43, 627-637; doi:I0.1038/npp.2017.225; published online 25 October 2017
\end{abstract}

\section{INTRODUCTION}

Among the many hormones, neuropeptides, and neurotransmitters that influence feeding behavior, the glucagonlike peptide-1 (GLP-1) system stands out as a key regulator of physiological and behavioral processes involved in glycemic and food intake control (Grill and Hayes, 2012). Within the central nervous system (CNS), GLP-1 is produced from the preproglucagon (PPG) neurons of the caudal nucleus tractus solitarius (NTS). These PPG neurons project widely throughout the CNS (Alhadeff et al, 2012; Dossat et al, 2011; Kanoski et al, 2016), suggesting that GLP-1 receptor (GLP-1R) populations that are distributed across the neuraxis (Merchenthaler et al, 1999) are activated by centrally produced GLP-1, either through direct projections of PPG neurons or through volume transmission (Alhadeff et al, 2012; Dossat et al, 2011; Hsu et al, 2015; Kanoski et al, 2016). However, the functional role of each of

\footnotetext{
*Correspondence: Dr MR Hayes, Department of Psychiatry, University of Pennsylvania, Center for Neurobiology and Behavior, 125 S. 3 I st St, Philadelphia, PA 19104, USA, Tel: + 215573 6070, Fax: + 215573 204I,E-mail: hayesmr@mail.med.upenn.edu

${ }^{4}$ Current address: Department of Exercise and Nutrition Sciences, State University of New York at Buffalo, Buffalo, NY, USA

Received 3 July 2017; revised 12 September 2017; accepted 13 September 2017; accepted article preview online 18 September 2017
}

these GLP-1R populations in mediating the intake suppressive effects of exogenous GLP-1R agonists and/or endogenous NTS-derived GLP-1 remains largely unresolved. This gap in our knowledge is of clinical significance, as FDAapproved GLP-1R agonists penetrate into the CNS to activate central GLP-1Rs to suppress food intake (Hayes et al, 2011a; Kanoski et al, 2016; Reiner et al, 2016).

The lateral dorsal tegmental nucleus (LDTg) of the mesopontine tegmentum expresses GLP-1Rs (Merchenthaler et al, 1999), receives axonal projections from hindbrain, hypothalamic, and midbrain nuclei (Cornwall et al, 1990), and is anatomically positioned to serve as a potential hub modulating energy balance and motivated behavior (Reiner et al, 2017). The LDTg is classically associated with modulation of ventral tegmental area (VTA) dopamine cell firing as well as effects on mesolimbic and basal ganglia neurotransmission and related behaviors, such as motivated behavior and psychostimulant-induced locomotion (Alderson et al, 2005; Blaha et al, 1996; Dobbs and Cunningham, 2014; Forster et al, 2002; Laviolette et al, 2000; Lodge and Grace, 2006; Omelchenko and Sesack, 2005; Steidl et al, 2015). As the literature has implicated the mesolimbic system in feeding over recent years (DiLeone et al, 2012; Narayanan et al, 2010), it is important to examine the role of upstream modulators of the mesolimbic system, such as the LDTg. Indeed, neuropeptide systems have been shown to act 
in the LDTg to modulate energy balance, establishing a role for the LDTg in food intake and meal size control, body weight regulation, and appetitive behavior (Dickson et al, 2011, 2010; Jerlhag et al, 2012; Reiner et al, 2017). The NTS projects to the LDTg, and critically, these projections originate at the same rostral-caudal level as NTS PPG neurons (Cornwall et al, 1990). This suggests that a portion of these LDTg-projecting NTS neurons is GLP-1-positive and may provide an endogenous central source of GLP-1 to the LDTg. As the NTS receives input from the gastrointestinal tract via the vagus nerve (Grill and Hayes, 2009, 2012), these anatomical findings provide the intriguing possibility of a potential role of gut-LDTg signaling through an NTS-to-LDTg GLP-1 pathway.

Given that the LDTg expresses GLP-1R and integrates information from peripherally and centrally derived energy status signals to affect energy balance (Dickson et al, 2011, 2010; Jerlhag et al, 2012; Reiner et al, 2017), we examined the hypothesis that GLP-1R signaling in the LDTg is both pharmacologically and physiologically relevant for energy balance control. Our data establish a novel role of GLP-1 signaling in the LDTg for energy balance control and provide further anatomical and behavioral evidence that the LDTg is a potential hub mediating ingestive behavior.

\section{MATERIALS AND METHODS}

\section{Animals \\ Male Sprague-Dawley rats (310-325 g upon arrival; Charles River or Envigo) were individually housed in hanging wire cages ( $12 \mathrm{~h}$ light/dark cycle) and given ad libitum chow (Purina LabDiet 5001) and water. All procedures were approved by the Institutional Animal Care and Use Committee at the University of Pennsylvania and at the University of Southern California and were performed according to the National Institutes of Health guidelines.}

\section{Drugs}

Exendin-4 (Ex-4; Bachem, Torrance, CA) was dissolved in artificial cerebrospinal fluid (aCSF; Harvard Apparatus) for central injections and $0.9 \%$ sterile saline for peripheral injections. Exendin-(9-39) (Ex-9; Bachem) was dissolved in aCSF. Fluorescent exendin-4 (FLEX; Anaspec) was dissolved in $99.97 \%$ saline and $0.03 \%$ aCSF.

\section{Stereotaxic Surgery}

Animals were anesthetized with an intramuscular (IM) injection of a cocktail (KAX) composed of ketamine (90 mg/kg), acepromazine $(0.64 \mathrm{mg} / \mathrm{kg})$, and xylazine $(2.7 \mathrm{mg} / \mathrm{kg})$ and placed in a stereotaxic apparatus. Bilateral guide cannulae (26 gauge; Plastics One, Roanoke, VA) targeting $2.5 \mathrm{~mm}$ dorsal to the LDTg (according to Paxinos and Watson, 2005); coordinates: $\pm 0.5 \mathrm{~mm}$ lateral to midline, $8.7 \mathrm{~mm}$ posterior to bregma, $4.1 \mathrm{~mm}$ ventral to skull) were implanted and affixed to the skull with bone screws and dental cement. Analgesia (meloxicam, $2 \mathrm{mg} / \mathrm{kg}$ ) was administered to all animals following surgery. Rats were allowed $\sim 1$ week recovery from surgery before the beginning of an experiment. For microinjections, we used a Plastics One 33 gauge microinjector that extended an additional $2.5 \mathrm{~mm}$ beyond the length of the cannula (total depth: $6.6 \mathrm{~mm}$ ventral to skull). Microinjectors were attached to pump-mounted syringes, and pressure injections were $100 \mathrm{nl}$ volume (or $200 \mathrm{nl}$ where noted) at a speed of $100 \mathrm{nl} / \mathrm{s}$. LDTg injection placements were verified histologically post mortem by intraparenchymal injections of pontamine sky blue $(100 \mathrm{nl})$. We limited each animal to no more than six microinjections for each experiment and there was minimal LDTg damaged tissue in our post-mortem analysis. Animals with cannula placements outside of the LDTg were eliminated from analyses. Maps of all cannula placements and behavioral data from animals with incorrect cannula placements can be found in the Supplementary Data.

\section{Immunohistochemistry}

GLP-1 axon labeling in the LDTg. Immunohistochemical labeling of GLP-1 axons in the LDTg was performed as previously described (Hsu et al, 2015). Briefly, rats $(n=4)$ were transcardially perfused and brains were removed and sectioned coronally at $30 \mu \mathrm{m}$. LDTg-containing sections were incubated for $60 \mathrm{~h}$ at $4{ }^{\circ} \mathrm{C}$ in primary antibody for rabbit anti-GLP-1 (1:2000, T-4363; Peninsula Labs, San Carlos, CA) in potassium phosphate-buffered saline (KPBS) with $0.1 \%$ Triton X-100. Following primary antibody incubation, sections were incubated overnight at $4{ }^{\circ} \mathrm{C}$ with biotinylated donkey anti-rabbit (Jackson Immunoresearch) in KPBS with $0.1 \%$ Triton X-100. Following a KPBS rinse, sections were incubated with KPBS with $\mathrm{ABC}$ reagent (1:1000, reagent $\mathrm{A}$ and $\mathrm{B}$ from $\mathrm{ABC}$ Elite kit; Vector Labs) for $4 \mathrm{~h}$. Following further KPBS washes, sections were reacted for $10 \mathrm{~min}$ in KPBS containing $0.005 \% \mathrm{H}_{2} \mathrm{O}_{2}$ and $0.05 \%$ 3,3'-diaminobenzidine (DAB). Following final washes, the sections were mounted on glass slides, air-dried and coverslipped with DePeX. Photomicrographs were taken with a digital camera mounted to a microscope (Nikon 80i) equipped for darkfield illumination to view the DAB-label as a 'gold-ish' color.

Retrograde tracing of GLP-1 NTS-to-LDTg projections. Rats $(n=3)$ were anesthetized with a surgical dose of KAX and received a unilateral LDTg injection of $100 \mathrm{nl}$ of $0.25 \%$ cholera toxin B subunit (CTB; \#104; List Laboratories) over $2 \mathrm{~s}$, according to the following coordinates (according to Paxinos and Watson, 2005): $0.6 \mathrm{~mm}$ lateral to midline, $8.7 \mathrm{~mm}$ posterior to bregma, $4.5 \mathrm{~mm}$ ventral to skull; microinjector aimed $7.0 \mathrm{~mm}$ ventral to skull. After the injection, the microinjector was left in place for $10 \mathrm{~min}$ before being removed and the incision was sutured. Rats were perfused 7 days later. Following removal, brains were postfixed in $4 \%$ paraformaldehyde for $6 \mathrm{~h}$ and cryoprotected in $20 \%$ sucrose in $0.1 \mathrm{M} \mathrm{PBS}$ at $4{ }^{\circ} \mathrm{C}$ for $2-3$ days. Coronal brain sections $(30 \mu \mathrm{m})$ encompassing the NTS or LDTg were sliced and stored in cryoprotectant until processing. Sections were washed in 50\% ethanol for $30 \mathrm{~min}$, rinsed in PBS, and then incubated in $1 \%$ sodium borohydride for $20 \mathrm{~min}$. Following a PBS wash, sections were then blocked in PBS with $5 \%$ normal donkey serum and $0.2 \%$ Triton-X at room temperature for $1 \mathrm{~h}$. NTS-containing sections were incubated overnight at room temperature in the following primary antibodies: mouse anti-CTB (1:1000, ab62429; Abcam) and rabbit anti-GLP-1 (1:1000). LDTg-containing sections were 
incubated overnight in the mouse anti-CTB antibody. Following a PBS rinse, sections were incubated for $2 \mathrm{~h}$ in the appropriate donkey anti-primary antibody species Alexa Fluor 488 and 594 antibodies (Jackson Immunoresearch). All antibody incubations were performed in blocking solution. Sections were mounted on slides and visualized with a Leica SP5 X confocal microscope using the 488 and 594 laser lines with $\mathrm{a} \times 20$ objective. All images were collected sequentially to avoid contamination of signals from other fluorophores. At least six NTS-containing sections from 14.16 to $14.64 \mathrm{~mm}$ posterior to bregma were used to quantify the number of CTB and PPG neurons.

Fluorescent exendin-4 labeling in the LDTg. To provide anatomical evidence that a systemically administered GLP-1R agonist penetrates into the brain and binds to cells within the LDTg, rats $(n=5)$ received an IP injection of fluorescent exendin-4 (FLEX; $3 \mu \mathrm{g} / \mathrm{kg}$ ) at the onset of the dark cycle. Rats were food deprived for $1 \mathrm{~h}$ prior to dark cycle onset and food remained unavailable. Three hours later, rats were anesthetized with an IM injection of the surgical dose of KAX and transcardially perfused with $0.1 \mathrm{M}$ PBS followed by $4 \%$ paraformaldehyde in $0.1 \mathrm{M}$ PBS. Brains were removed, postfixed, sectioned, and tissue was blocked as described above. LDTg-containing sections were incubated overnight in primary antibodies (rabbit anti-glial fibrillary acidic protein (GFAP; 1:2000, Z0334; Dako/Agilent) and mouse anti-NeuN (1:1000, MAB377; Millipore)) in blocking solution. Following a PBS rinse, sections were incubated in appropriate donkey Alexa Fluor 594 and 647 secondary antibodies (Jackson Immunoresearch) at room temperature. Sections were mounted on slides and visualized with a Leica SP5 X confocal microscope using the 488, 594, and 633 laser lines with a $\times 63$ oil immersion objective. FLEX was visualized in the LDTg using the 488 laser line. All images were collected sequentially to avoid contamination of signals from other fluorophores. Images are represented as a maximal z-stack projection with Fiji 2.0 or as a $3 \mathrm{D}$ rotational video with Imaris (8.4.1).

\section{Behavioral Testing}

General procedures. Following a week of handling prior to surgery, rats were again handled daily and habituated to the injection procedure for five consecutive days prior the start of the experiment. Drug injections were performed immediately prior to the onset of the dark cycle unless otherwise specified. For experiments measuring ad libitum food intake, food weights were measured at $1,3,6$, and $24 \mathrm{~h}$ after injection, unless otherwise noted. Food weights were recorded to the nearest $0.1 \mathrm{~g}$, accounting for spillage. Body weight was measured at 0 and $24 \mathrm{~h}$ after injection. Treatments were assigned using a within-subject counterbalanced design, with at least $72 \mathrm{~h}$ between injections. Each behavioral experiment was conducted in a separate cohort of rats.

Intra-LDTg exendin-4 dose-response experiments. To assess the dose-response effects of LDTg GLP-1R activation on food intake and body weight, rats $(n=13)$ received unilateral LDTg injections of Ex-4 (0, 0.025, $0.05 \mu \mathrm{g} ; 100 \mathrm{nl}$
aCSF) immediately before the onset of the dark cycle. These doses of Ex-4 were selected based on previous dose-response experiments in the NTS, VTA, and nucleus accumbens (NAc) (Alhadeff et al, 2012; Hayes et al, 2008). Food intake and body weight were measured. Post-mortem analyses revealed that cannula accurately targeted the LDTg in 10 rats and are included in the final behavioral data for this experiment (Figure 1a and b). Cannula outside of the LDTg were observed in three rats, and behavioral data from these rats are graphed in Supplementary Figure S1A and B. All cannula placements for this experiment are shown in a schematic in Supplementary Figure S1C (based on Paxinos and Watson, 2007).

To determine if nausea/malaise contributes to the intake suppression following GLP-1R activation in the LDTg, pica, the intake of non-nutritive substances and a well-established model of nausea/malaise (Andrews and Horn, 2006; Kanoski et al, 2012a; Mitchell et al, 1977; Takeda et al, 1993; Yamamoto et al, 2002), was measured. Rats $(n=12)$ were given access to kaolin clay (Research Diets) for $\sim 1$ week prior to the experiment. Chow and kaolin clay intake were measured at $24 \mathrm{~h}$ after unilateral LDTg injection of exendin-4 (0, 0.025, $0.05 \mu \mathrm{g} ; \quad 100 \mathrm{nl}$ aCSF). Post-mortem analyses revealed that cannula accurately targeted the LDTg in eight rats and are included in the final behavioral data for this experiment (Figure $1 \mathrm{c}$ and $\mathrm{d}$ ). Cannula outside of the LDTg were observed in four rats, and behavioral data from these rats are graphed in Supplementary Figure S2A and B. All cannula placements for this experiment are shown in a schematic in Supplementary Figure S2C.

For meal pattern experiments, rats $(n=13)$ were housed in a custom-made automated feedometer system consisting of hanging wire cages with access to a food cup on an electronic scale (Hayes et al, 2011b; Kanoski et al, 2012b; MietlickiBaase et al, 2013), which records the weight of food cups every $10 \mathrm{~s}$ through computer software (Labview). A meal was defined as at least $0.25 \mathrm{~g}$ of food ingested with $10 \mathrm{~min}$ or more between feeding bouts (Hayes et al, 2011b; Kanoski et al, 2012b; Mietlicki-Baase et al, 2013). Meal patterns were assessed at 12 and $24 \mathrm{~h}$ post LDTg injection of exendin-4 (0, $0.025,0.05 \mu \mathrm{g} ; 100 \mathrm{nl}$ aCSF). Post-mortem analyses revealed that cannula accurately targeted the LDTg in 10 rats and are included in the final behavioral data for this experiment (Figure 1e and f). Cannula outside of the LDTg were observed in three rats, and behavioral data from these rats are graphed in Supplementary Figure S3A and B. All cannula placements for this experiment are shown in a schematic in Supplementary Figure S3C.

LDTg GLP-1R blockade feeding experiments. To determine the physiological role of GLP-1R signaling in the LDTg for energy balance control, we assessed the dose response of GLP-1R blockade in the LDTg on food intake and body weight gain. Rats $(n=11)$ received unilateral LDTg injections of the competitive GLP-1R antagonist Ex-9 (0, 10, $20 \mu \mathrm{g}$; $200 \mathrm{nl}$ aCSF; doses chosen based on Hayes et al, 2009) immediately before the onset of the dark cycle. Food intake and body weight were measured. Post-mortem analyses revealed that cannula accurately targeted the LDTg in nine rats and are included in the final behavioral data for this 
a

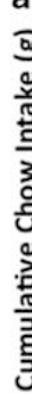

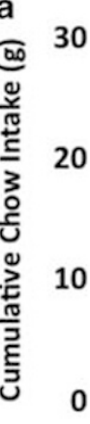

$\square$ aCSF

\section{ㅁ $0.025 \mu \mathrm{g} \mathrm{Ex}-4$}

D $0.05 \mu \mathrm{g} \mathrm{Ex-4}$
-

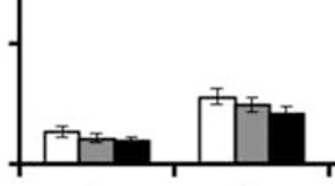

3

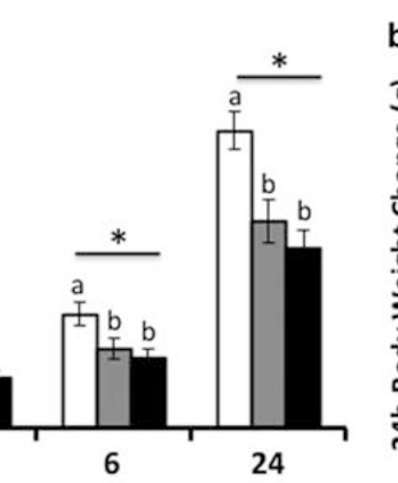

24 b

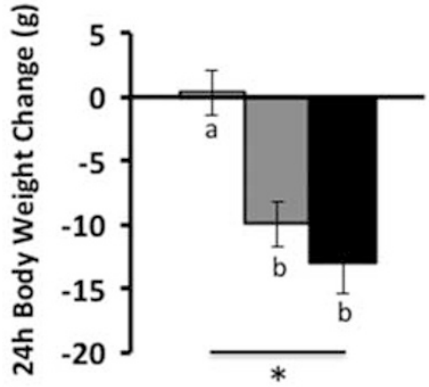

Time (h)

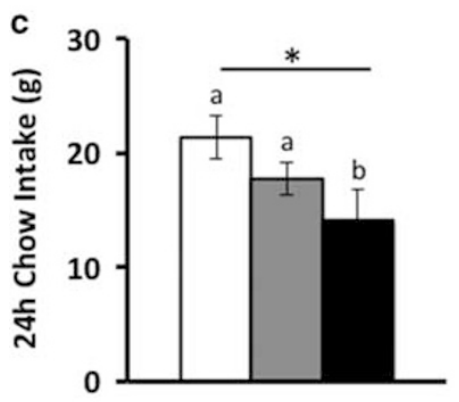

e

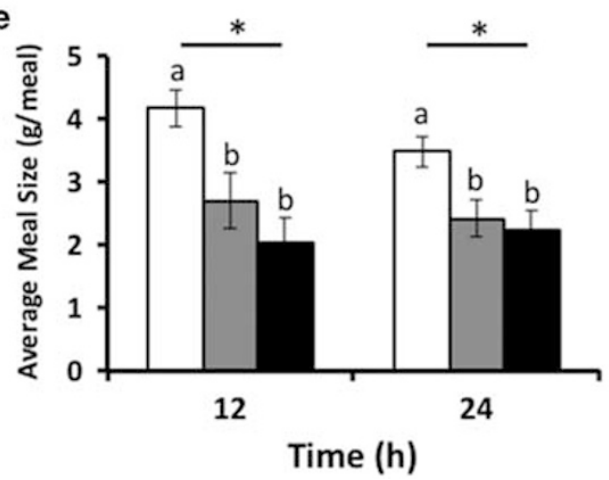

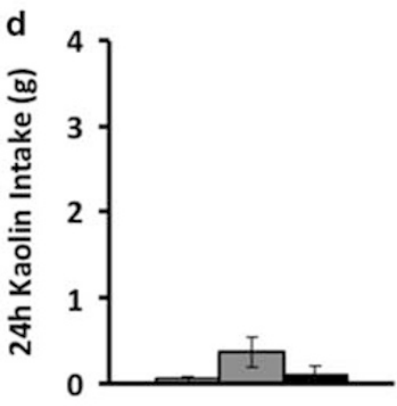

f

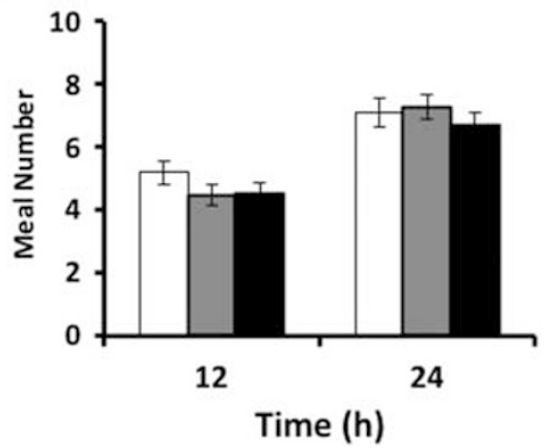

Figure I Intra-LDTg GLP-IR activation suppresses food intake through a decrease in average meal size and independent of malaise. The GLP-IR agonist Ex-4 was unilaterally injected in the LDTg in a counterbalanced within-subjects design $(n=10)$ at the onset of the dark cycle using the following doses: 0 (aCSF), 0.025 , or $0.05 \mu \mathrm{g}$. These doses of Ex-4 suppress food intake at 6 and $24 \mathrm{~h}$ (a) and also reduce $24 \mathrm{~h}$ body weight change (b). A schematic map of cannula placements for ( $\mathrm{a}$ and $\mathrm{b}$ ) can be found in Supplementary Figure SIC. To determine if LDTg GLP-IR activation produces nausea/malaise, kaolin intake was measured following unilateral Ex-4 LDTg administration. In a separate cohort of rats $(n=8)$, intra-LDTg Ex-4 decreases $24 \mathrm{~h}$ food intake (c) but has no effect on $24 \mathrm{~h}$ kaolin intake (d). A schematic map of cannula placements for (c and d) can be found in Supplementary Figure S2C. Meal patterns were analyzed in a third cohort of rats $(n=10)$. Ex-4 decreases 12 and $24 \mathrm{~h}$ average meal size (e) but had no effect on meal number ( $f$ ). A schematic map of cannula placements for (e and $f$ ) can be found in Supplementary Figure S3C. * indicates significance by repeated measures ANOVA ( $p<0.05)$. Different letters are significantly different from each other $(p<0.05)$ according to post hoc tests. The key in (a) applies to all panels. aCSF, artificial cerebrospinal fluid; GLP-IR, glucagon-like peptide-I receptor; LDTg, lateral dorsal tegmental nucleus.

experiment (Figure 3a and b). Cannula outside of the LDTg were observed in two rats (data not shown), making statistical analyses impossible. All cannula placements for this experiment are shown in a schematic in Supplementary Figure S4.

To examine whether GLP-1R blockade in the LDTg attenuates the intake-suppressive effects of voluntary ingestion of a nutritionally complete preload, rats $(n=8)$ were trained to drink $9 \mathrm{ml}$ of vanilla Ensure (Abbott Nutrition) in 15 min just prior to dark cycle onset. Immediately following the ingestion of Ensure, rats received unilateral LDTg injections of Ex-9 (0, $10 \mu \mathrm{g} ; 100 \mathrm{nl}$ aCSF; dose chosen to be subthreshold for effect alone on feeding when administered in the LDTg) in a counter-balanced within subjects design. Injections were separated by at least $48 \mathrm{~h}$ and following training, rats only received access to Ensure on injection days. Chow was removed during the 15 min Ensure access period and was given back immediately following the injection. Chow intake was measured at $0.5,1,1.5,2,4$, and $24 \mathrm{~h}$ after injection, and body weight was measured at 0 and $24 \mathrm{~h}$ after injection. Post-mortem analyses revealed that cannula accurately targeted the LDTg in five rats and are included in the final behavioral data for this experiment (Figure $3 \mathrm{c}$ and $\mathrm{d}$ ). Cannula outside of the LDTg were 
observed in three rats, and behavioral data from these rats are graphed in Supplementary Figure S5A and B. All cannula placements for this experiment are shown in a schematic in Supplementary Figure S5C.

To examine whether acute pharmacological blockade of LDTg GLP-1R attenuates the intake-suppressive effects of systemic Ex-4 administration, we injected rats $(n=18)$ with Ex-9 (0, $10 \mu \mathrm{g} ; 100 \mathrm{nl}$ aCSF; dose chosen to be subthreshold for effect alone on feeding when administered in the LDTg) unilaterally in the LDTg $1 \mathrm{~h}$ prior to the onset of the dark cycle. Fifteen minutes prior to the onset of the dark cycle, rats were injected systemically with Ex-4 $(3 \mu \mathrm{g} / \mathrm{kg}$, IP), and subsequent food intake was measured. Post-mortem analyses revealed that cannula accurately targeted the LDTg in nine rats, which are included in the final behavioral data for this experiment (Figure 4c). Cannula outside of the LDTg were observed in nine rats, and behavioral data from these rats are graphed in Supplementary Figure S6A. All cannula placements for this experiment are shown in a schematic in Supplementary Figure S6B.

\section{Statistical Analyses}

All data are represented as mean \pm SEM with the $\alpha$-level set to $p=0.05$. Statistical analyses were performed using Statistica (Statsoft). For behavioral studies, binned data were analyzed using separate repeated measures one-way ANOVAs that accounted for the within-subjects experimental design. Statistically significant effects were probed using Student-Neuman-Keuls post hoc analyses except when noted.

\section{RESULTS}

\section{LDTg GLP-1R Activation Reduces Cumulative Chow Intake and Body Weight}

As the LDTg expresses GLP-1R mRNA and receives projections from the NTS, a region that contains GLP-1producing neurons (Cornwall et al, 1990; Merchenthaler et al, 1999), we first tested whether activation of GLP-1R in the LDTg suppresses food intake and body weight. Unilateral administration of Ex-4 in the LDTg decreases chow intake at 6 and $24 \mathrm{~h}$ after injection compared with aCSF vehicle treatment $\left(n=10, \mathrm{~F}_{2,18} \geqslant 5.64, p<0.05\right.$; Figure 1a). Post hoc analyses reveal that both doses of Ex-4 significantly suppress chow intake at 6 and $24 \mathrm{~h}$ post-injection ( $p<0.05$ compared with vehicle). Intra-LDTg Ex-4 also dose-dependently decreases $24 \mathrm{~h}$ body weight change $\left(\mathrm{F}_{2,18}=11.16, p<0.001\right.$; Figure 1b), with post hoc analyses showing a significant effect with the highest dose of Ex-4 $(p<0.05$ compared with vehicle). In rats with cannula placements outside of the LDTg $(n=3)$, there is no significant effect of Ex-4 on food intake or on $24 \mathrm{~h}$ body weight change $\left(\mathrm{F}_{2,4} \leqslant 0.99, p>0.44\right.$; Supplementary Figure S1A and B).

\section{LDTg GLP-1R Activation Does Not Produce Malaise}

To determine if the intake suppression following LDTg GLP-1R activation is driven by nausea/malaise, we injected the same doses of Ex-4 into the LDTg and measured pica. Intra-LDTg significantly decreases $24 \mathrm{~h}$ chow intake compared with vehicle $\left(n=9, \quad F_{2,14}=3.91, \quad p<0.05\right.$; Figure 1c) with post hoc analyses showing that the higher dose of Ex-4 significantly reduces chow intake $(p<0.05)$. Importantly, intra-LDTg has no effect on $24 \mathrm{~h}$ kaolin intake $\left(\mathrm{F}_{2,14}=2.00, p<0.05\right.$; Figure $\left.1 \mathrm{~d}\right)$, suggesting that nausea/ malaise is not driving the hypophagia following intra- $\mathrm{LDTg}$ GLP-1R activation. In rats $(n=4)$ with cannula placements outside of the LDTg, there is no significant effect of Ex-4 on $24 \mathrm{~h}$ food intake or $24 \mathrm{~h}$ kaolin intake $\left(\mathrm{F}_{2,6} \leqslant 1.21, p>0.36\right.$; Supplementary Figure S2A and B).

\section{LDTg GLP-1R Activation Suppresses Average Meal Size}

Meal pattern analyses show that unilateral injection of Ex-4 in the LDTg significantly decreases average meal size at 12 and $24 \mathrm{~h}\left(n=10, \mathrm{~F}_{2,18}>5.73, p<0.05\right.$; Figure 1e), but has no effect on meal number at either time point $\left(\mathrm{F}_{2,18}<0.08\right.$, $p>0.9$; Figure 1f). Post hoc analyses reveal that both doses of Ex-4 suppress average meal size at 12 and $24 \mathrm{~h}$ post-injection $(p<0.05)$. These data suggest that LDTg GLP-1R activation reduces food intake predominantly through a reduction in average meal size with minimal effects on meal number. In rats $(n=3)$ with cannula placements outside of the LDTg, there is no significant effect of Ex-4 on meal size or meal number $\left(\mathrm{F}_{2,4} \leqslant 1.26, p>0.37\right.$; Supplementary Figure S3A and B).

\section{GLP-1-Expressing Axon Terminals are Present in the LDTg, and NTS PPG Neurons Project to the LDTg}

Central GLP-1 is produced by PPG neurons in the NTS, which project widely throughout the CNS, including to the VTA and NAc (Alhadeff et al, 2012; Grill and Hayes, 2012; Hayes et al, 2014; Kanoski et al, 2016; Vrang et al, 2003). We therefore performed IHC analyses to test whether GLP-1 axons are also present in the LDTg. Indeed, GLP-1immunopositive axons are present in the LDTg (Figure 2a and b; approximately $8.64 \mathrm{~mm}$ posterior to bregma).

To directly determine the endogenous source of GLP-1 axons projecting into the $\mathrm{LDTg}$, the retrograde tracer CTB was injected into the LDTg $(n=3)$ and animals were killed seven days later. Retrograde labeling of CTB shows that $5.56 \pm 1.05 \%$ of CTB-immunopositive NTS neurons colocalize with PPG and $4.24 \pm 1.00 \%$ of PPG-immunopositive neurons colocalize with CTB (see Figure $2 c-e$ for representative images; see Table 1 for complete quantification data). A representative image shows that the CTB injection site from this same animal is within the LDTg (Figure 2f; approximately $8.52 \mathrm{~mm}$ posterior to bregma). We did not observe co-localization between CTB and PPG in the reticular formation (data not shown) and therefore limited our quantification to the NTS GLP-1 neurons. These data provide evidence that a portion of NTS PPG neurons project to the LDTg. Collectively, IHC data showing the presence of GLP-1-immunopositive fibers in the LDTg and that NTS PPG neurons project to the LDTg provide anatomical evidence of a physiological role of endogenous GLP-1R signaling in the LDTg. 

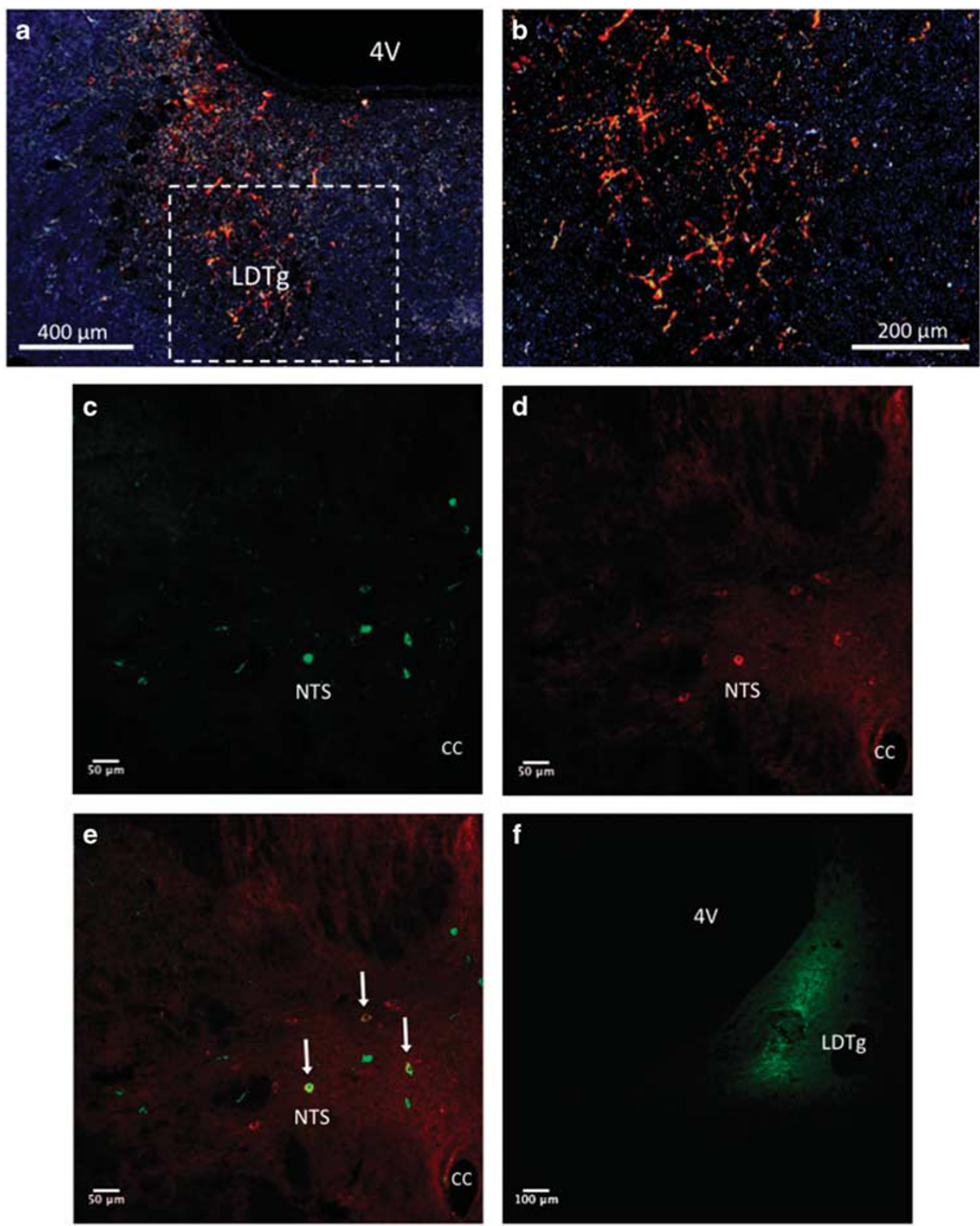

Figure 2 GLP-I-positive axons are present in the LDTg, and PPG neurons project from the NTS to the LDTg. Immunolabeling shows the presence of GLP-I-positive axons in the LDTg $(n=4)$. Representative images are shown in $(a, b)$. The white dashed-line box in $(a)$ indicates the field of view in (b). To determine if the NTS PPG neurons project to the LDTg, rats $(n=3)$ were unilaterally injected with the neural pathway tracer CTB in the LDTg. Seven days later, rats were perfused and their brains were processed. Within the NTS, $5.56 \pm 1.05 \%$ of retrogradely labeled CTB-positive neurons (green) colocalize with PPG-positive neurons (red). Representative images show CTB-positive neurons in green (c), PPG-positive neurons in red (d), and merged in (e). Panel ( $f$ ) shows a representative CTB LDTg injection site. The white arrows in (e) indicate co-localization (yellow). 4V, 4th ventricle; cc, central canal; CTB, cholera toxin B; GLP-IR, glucagon-like peptide-I receptor; LDTg, lateral dorsal tegmental nucleus; NTS, nucleus tractus solitarius; PPG, preproglucagon.

\section{LDTg GLP-1R Blockade Increases Food Intake}

Intra-LDTg GLP-1R blockade with the competitive GLP-1R antagonist Ex-9 significantly increases food intake $(n=9$, $\mathrm{F}_{2,16}=3.64, p<0.05$; Figure $\left.3 \mathrm{a}\right)$ at $24 \mathrm{~h}$ with no effect on body weight $\left(\mathrm{F}_{2,16}=0.19, p=0.8\right.$; Figure $\left.3 \mathrm{~b}\right)$. Importantly, the $10 \mu \mathrm{g}$ dose of Ex-9 has no effect on food intake compared with aCSF vehicle $(p>0.05)$, but the $20 \mu \mathrm{g}$ dose of Ex-9 significantly increases food intake at $24 \mathrm{~h}(p<0.05)$. These data suggest that GLP-1R signaling in the LDTg is physiologically relevant for food intake control. Only two rats had cannula placements outside of the LDTg in this experiment (data not shown), making statistical analysis impossible.

\section{LDTg GLP-1R Blockade Attenuates the Intake Suppressive Effects of a Self-Ingested Preload}

To determine the potential role of a gut-to-NTS-to-LDTg GLP-1 pathway in mediating food intake, we next examined if LDTg GLP-1R blockade can attenuate the intake suppressive effects of a voluntary ingested gastric preload (9 ml Ensure) gastric distension. To avoid competing 
Table I Quantification Data of CTB-Positive and PPG-Positive Neurons in the NTS

\begin{tabular}{|c|c|c|c|c|c|c|c|c|}
\hline Rat & Section & Atlas level & $\begin{array}{l}\text { Distance from } \\
\text { bregma (mm) }\end{array}$ & $\begin{array}{l}\text { \# CTB+ } \\
\text { neurons }\end{array}$ & $\begin{array}{l}\text { \# PPG+ } \\
\text { neurons }\end{array}$ & $\begin{array}{c}\text { \# CTB+ \& PGG+ } \\
\text { neurons }\end{array}$ & $\begin{array}{l}\% \text { CTB+ that } \\
\text { are PPG+ }\end{array}$ & $\begin{array}{c}\text { \%PPG+ that } \\
\text { are CTB+ }\end{array}$ \\
\hline \multirow[t]{4}{*}{ I } & I & Caudal to obex & -14.64 & 26 & 21 & 2 & 7.69 & 9.52 \\
\hline & 3 & Obex & -14.40 & 32 & 19 & 0 & 0 & 0 \\
\hline & 4 & Obex & -14.40 & 24 & 21 & 2 & 8.33 & 9.52 \\
\hline & 7 & Area postrema & -14.16 & 43 & 25 & 4 & 9.30 & 16.00 \\
\hline \multirow[t]{5}{*}{2} & । & Caudal to obex & -14.64 & 25 & 17 & । & 4.00 & 5.88 \\
\hline & 2 & Caudal to obex & -14.52 & 18 & 29 & 0 & 0.00 & 0.00 \\
\hline & 3 & Caudal to obex & -14.52 & 7 & 24 & 0 & 0.00 & 0.00 \\
\hline & 7 & Obex & -14.40 & 9 & 33 & I & $|1.1|$ & 3.03 \\
\hline & 8 & Area postrema & -14.28 & 9 & 33 & I & 11.11 & 3.03 \\
\hline \multirow[t]{7}{*}{3} & I & Caudal to obex & -14.64 & 17 & 15 & I & 5.88 & 6.67 \\
\hline & 2 & Caudal to obex & -14.52 & 17 & 15 & I & 5.88 & 6.67 \\
\hline & 3 & Obex & -14.40 & 19 & 28 & I & 5.26 & 3.57 \\
\hline & 4 & Obex & -14.40 & 17 & 15 & I & 5.88 & 6.67 \\
\hline & 5 & Obex & -14.40 & 12 & 26 & 0 & 0 & 0 \\
\hline & 6 & Obex & -14.40 & 23 & 35 & 0 & 0 & 0 \\
\hline & 7 & Area postrema & -14.16 & 16 & 27 & I & 6.25 & 3.70 \\
\hline
\end{tabular}

At least seven representative sections from the NTS of three LDTg-CTB injected animals were used to quantify the number of CTB-positive neurons, the number of PPG-positive neurons, and the number of neurons that were immunopositive for both markers.

orexigenic and anorectic behavioral responses, we intentionally used a dose of Ex-9 that is subthreshold for an effect on feeding when delivered unilaterally in the LDTg. GLP-1R blockade in the LDTg significantly attenuates the intake suppressive effects of gastric distension at 2 and $24 \mathrm{~h}$ postinjection $\left(n=5, \quad \mathrm{~F}_{1,4}>8.76, \quad p<0.05 ;\right.$ Figure $\left.3 \mathrm{c}\right)$ and approaches significance at $4 \mathrm{~h}\left(\mathrm{~F}_{1,4}=5.98, p=0.07\right)$. IntraLDTg Ex-9 also significantly increases body weight compared with aCSF vehicle treatment $\left(\mathrm{F}_{1,4}=9.53, p<0.05\right.$; Figure $3 \mathrm{~d})$. These data show that LDTg GLP-1R blockade can attenuate the intake suppressive effects of gastric distension, suggesting physiological relevance of either direct or indirect gut-to-LDTg signaling. In rats $(n=3)$ with cannula placements outside of the LDTg, there is no significant effect of Ex-9 on food intake or body weight $\left(\mathrm{F}_{1,2} \leqslant 3.93, p>0.19\right.$; Supplementary Figure S5A and B).

\section{Peripherally-Administered Fluorescent Exendin-4 is Juxtaposed with LDTg Neurons}

Given that GLP-1 mimetics are administered systemically in humans, we next evaluated whether a peripherally administered fluorescent GLP-1R agonist accesses the LDTg of rats. Indeed, $3 \mathrm{~h}$ following systemic administration, FLEX shows robust expression in the LDTg. Further, within the LDTg, FLEX predominantly juxtaposes with neurons and to a far-lesser degree there is weak association with GFAP-immunopositive astrocytes (Figures $4 \mathrm{a}$ and $\mathrm{b}$ and $3 \mathrm{~d}$ rotational video in Movie 1).

\section{LDTg GLP-1R Blockade Attenuates the Intake Suppressive Effects of a Systemically Administered GLP-1R Agonist}

Given anatomical data showing that a systemicallyadministered GLP-1R agonist accesses the LDTg, we next evaluated whether LDTg GLP-1R blockade attenuates the anorectic effects of systemic Ex-4. Systemic injection of Ex-4 significantly suppresses chow intake at $3 \mathrm{~h}(n=9$, main effect of Ex-4, $\left.\mathrm{F}_{1,8}=6.31, p<0.05\right)$ and approaches significance at $6 \mathrm{~h}$ (main effect of Ex-4, $\mathrm{F}_{1,8}=5.15, p=0.053$ ) after injection (Figure 4c). There is also a significant interaction between Ex-4 and Ex-9 at 3 and $6 \mathrm{~h}$ after injection $\left(\mathrm{F}_{1,8} \geqslant 6.44\right.$, $p<0.05)$; post hoc analyses show that pre-treatment with 

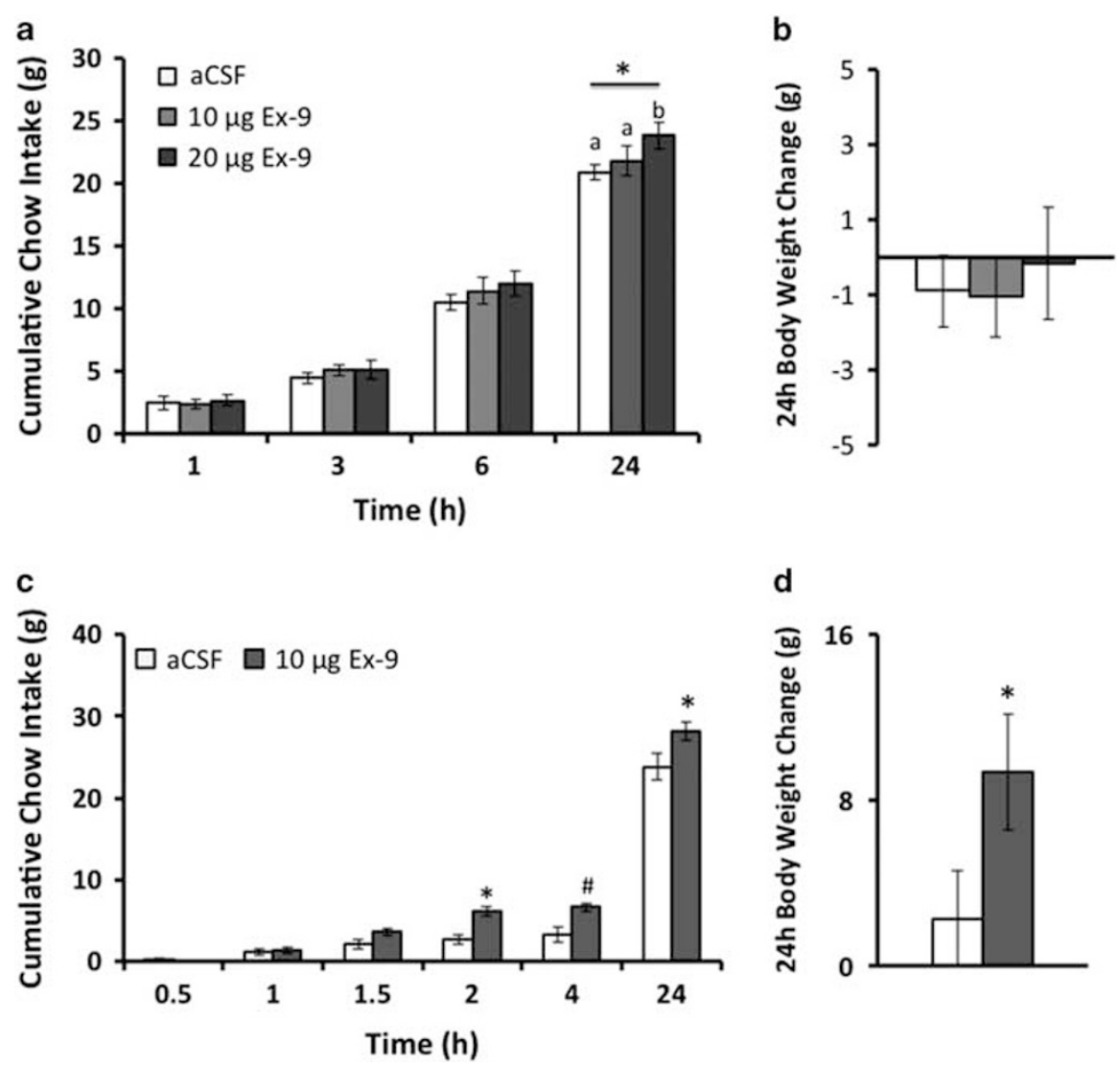

Figure 3 LDTg GLP-IR blockade increases food intake and attenuates the intake suppressive effects of a self-ingested preload. To determine the effect of GLP-IR blockade in the LDTg on food intake, the competitive GLP-IR antagonist Ex-9 was injected unilaterally in the LDTg in a counterbalanced withinsubjects design at the onset of the dark cycle using the following doses: 0 (aCSF), $10,20 \mu g ; 200 \mathrm{nl}(n=9)$. Only the highest dose of Ex-9 (20 $\mu \mathrm{g})$ increases food intake at $24 \mathrm{~h} \mathrm{(a)}$ and neither dose of Ex-9 had an effect on $24 \mathrm{~h}$ body weight change (b). A schematic map of cannula placements for (a,b) can be found in Supplementary Figure S4. To determine if LDTg GLP-IR blockade can attenuate the hypophagic effects of gastric distension, rats ( $n=5)$ drank 9 ml of Ensure in 15 min just prior to dark cycle onset and were subsequently given unilateral LDTg injections of Ex-9 (0 (aCSF), I0 Mg; I00 nl aCSF) in a withinsubjects design. Ex-9 significantly increases food intake at 2 and $24 \mathrm{~h}$ after injection (c) and body weight over $24 \mathrm{~h}$ (d). A schematic map of cannula placements for $(c, d)$ can be found in Supplementary Figure S5C. * indicates significance $(p<0.05)$ and \# indicates a trend for significance $(p=0.07)$ by repeated measures ANOVA. Different letters are significantly different from each other $(p<0.05)$ according to post hoc tests. The key in $(a)$ applies to $(b)$, and the key in $(c)$ also applies to (d). aCSF, artificial cerebrospinal fluid; GLP-IR, glucagon-like peptide-I receptor; LDTg, lateral dorsal tegmental nucleus.

intra-LDTg Ex-9 reverses the intake-suppressive effects of peripheral Ex-4 at 3 and $6 \mathrm{~h}$ post-injection $(p<0.05)$. Importantly, LDTg GLP-1R blockade alone (Ex-9/vehicle) does not significantly increase chow intake at any time point (all $p>0.05$ compared with vehicle/vehicle), though there is a significant main effect of Ex-9 at 3 and $6 \mathrm{~h}\left(\mathrm{~F}_{1,8} \geqslant 5.27\right.$, $p<0.05)$ driven by the $\mathrm{Ex}-9 / \mathrm{Ex}-4$ condition. These data show that intra-LDTg GLP-1R blockade attenuates the hypophagic effects of systemically administered Ex-4, suggesting the potential preclinical relevance of LDTg GLP-1R signaling. In rats $(n=9)$ with cannula placements outside of the LDTg, Ex-9 has no effect on the intake-suppressive effects of peripheral Ex-4 (main effect of Ex-4, $\mathrm{F}_{1,8}=9.85, p<0.05$; no main effect of Ex-9 or interaction between Ex-4 and Ex-9, $\mathrm{F}_{1,8} \leqslant 2.58, p>0.14$; Supplementary Figure S6A). Collectively, these behavioral data complement the anatomical data in Figure $4 \mathrm{a}$ and $\mathrm{b}$ and Movie 1 and highlight that peripherally administered Ex-4 accesses the LDTg in a functional capacity, suggesting the preclinical relevance of GLP-1R signaling in the LDTg for energy balance control.

\section{DISCUSSION}

To improve current obesity pharmacotherapies, research aimed at understanding neuroendocrine signals and the neurobiological substrates that control energy balance is required. Recently, much attention has been focused on the GLP-1 system, in particular the contribution of central GLP-1 signaling to energy balance control. Indeed, GLP-1R are widely expressed throughout the CNS and both endogenous central GLP-1 and exogenous long-lasting GLP-1R agonists, activate these central GLP-1Rs to reduce food intake and body weight (see Kanoski et al, 2016 for a review). Given that GLP-1R are expressed widely throughout the CNS (Merchenthaler et al, 1999), it is important to examine the specific brain regions that mediate the feeding effects of GLP-1R ligands. Here we provide evidence that GLP-1R signaling in the LDTg, a nucleus involved in energy balance control and motivated behaviors (Dickson et al, 2011, 2010; Lammel et al, 2012; Reiner et al, 2017; Schmidt et al, 2009), is both pharmacologically and physiologically involved in energy balance control. 

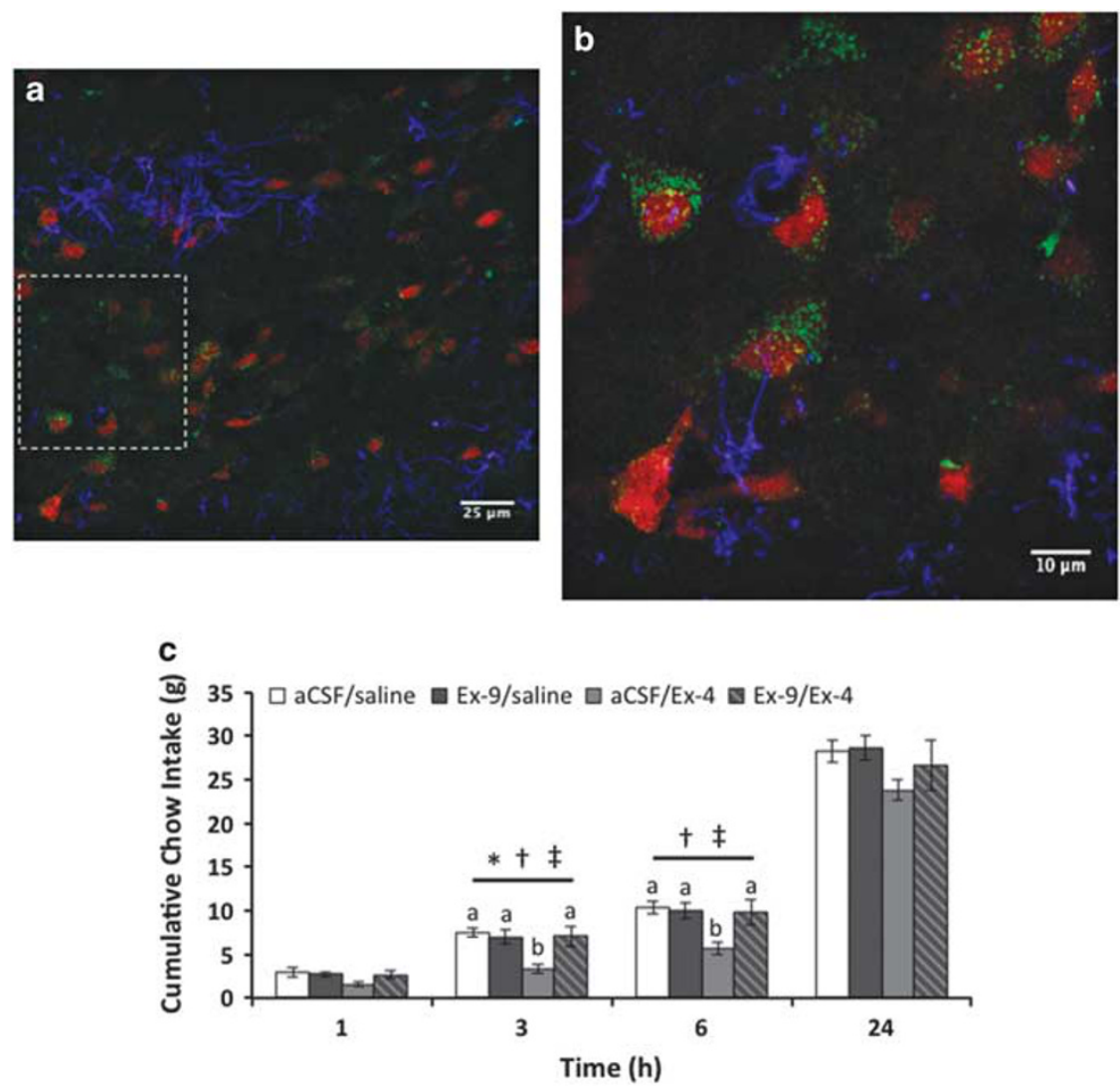

Figure 4 Systemically delivered GLP-IR agonists access the LDTg. To determine if peripherally administered Ex-4 penetrates the LDTg, we injected fluorescently tagged EX-4 (FLEX, $3 \mu g / \mathrm{kg}, I P)$, perfused the rats $(n=5) 3 \mathrm{~h}$ later, and processed the LDTg to visualize neurons, astrocytes, and FLEX. Peripherally administered FLEX (green) is juxtaposed with neurons (red) but minimally with astrocytes (blue) within the LDTg (a,b). $\times 63$ image in (a), and $\times 3$ optical zoom of $\times 63$ in (b). Dotted rectangle in (a) indicates field of view in (b) and in Movie I. To determine if GLP-IR blockade in the LDTg attenuates the hypophagic effects of peripheral Ex-4, the competitive GLP-IR antagonist Ex-9 was unilaterally injected in the LDTg $(n=9)$ at a dose subthreshold for an effect on feeding ( $10 \mathrm{Hg}$; vehicle, I $00 \mathrm{nl}$ aCSF) approximately I h prior to the onset of the dark cycle. Fifteen minutes prior to the onset of the dark cycle, rats were injected systemically with Ex-4 (0 (saline), 3 kg/kg). Ex-4 significantly suppresses food intake at $3 \mathrm{~h}(p<0.05)$ and approaches significance at $6 \mathrm{~h}$ post-injection $(p=0.06)$, and pre-treatment with Ex-9 reverses this intake suppression (c). A schematic map of accurate cannula placements for (c) can be found in Supplementary Figure S6B. * indicates significant main effect of Ex-4 $(p<0.05)$. $\dagger$ indicates a significant main effect of Ex-9 $(p<0.05)$. $\neq$ indicates a significant interaction between Ex-4 and Ex-9 $(p<0.05)$. Different letters are significantly different from each other $(p<0.05)$ according to post hoc tests. aCSF, artificial cerebrospinal fluid; GLP-IR, glucagon-like peptide-I receptor; IP, intraperitoneal; LDTg, lateral dorsal tegmental nucleus.

Current data show that intra-LDTg injection of the GLP-1R agonist Ex-4 decreases food intake and body weight, primarily through a reduction in average meal size. These data are consistent with existing literature showing that activation of GLP-1R in multiple nuclei suppresses food intake, predominately by a reduction in meal size (Dossat et al, 2013; Grill and Hayes, 2009, 2012; Hayes et al, 2008; Hsu et al, 2015; Scott and Moran, 2007; Terrill et al, 2016). The feeding effects of intra-LDTg Ex-4 occur at later time points compared with systemically delivered GLP-1R agonists. Our working hypothesis is that peripheral administration of a GLP-1R agonist activates multiple GLP-1R populations throughout the neuraxis to elicit intake suppression at early time points after injection. In contrast, the delayed intake suppression we observe with intra-LDTg Ex-4 is similar to the time course of the hypophagic effects after Ex-4 administration in a different single nucleus, such as the VTA, the NTS, and the ventral hippocampus (Alhadeff $e t a l$, 2012; Hayes et al, 2011b; Hsu et al, 2015).

Though peripheral and central administration of GLP-1 mimetics produces nausea/malaise (Kanoski et al, 2012a), our data show that direct LDTg GLP-1R activation does not produce pica, suggesting that GLP-1R signaling in the LDTg suppresses food intake independent of nausea/malaise. These data are consistent with data from other sites where GLP-1R activation suppresses food intake without producing nausea/ malaise (Alhadeff et al, 2012; Dossat et al, 2011; Hsu et al, 2015). Collectively, these behavioral data expand the known relevant CNS sites of action for GLP-1 signaling in mediating energy balance.

The current studies also support a physiological role for LDTg GLP-1R signaling in energy balance control. Acute unilateral blockade of LDTg GLP-1R increases food intake, and at a dose subthreshold for an effect on feeding, blockade of GLP-1R in the LDTg can attenuate the intake suppressive effects of a mixed macronutrient preload. Our data show that unilateral LDTg GLP-1R blockade is sufficient to increase food intake, but bilateral administration of intra-LDTg Ex-9 would likely be more effective at producing greater or perhaps earlier increases in food intake. Collectively, these data provide novel evidence of a physiological role for LDTg GLP-1R signaling in energy balance control and suggest that the LDTg putatively receives communication from the gut either through a humoral mechanism or more likely via a 
vagal-to-NTS-to-LDTg pathway. We also cannot rule out a potential role of nutrient signaling with the mixedmacronutrient Ensure preload in mediating the LDTg GLP-1R-dependent effects observed (Hayes et al, 2009). Given that gastric distension activates PPG neurons in the NTS (Hayes et al, 2009; Vrang et al, 2003), combined with current data here showing that NTS PPG neurons project to the LDTg, the collective set of data supports the hypothesis that LDTg GLP-1 signaling mediates, at least in part, the satiation signaling arising from meal ingestion via a vagalNTS-LDTg pathway. Indeed, IHC data show that GLP-1immunopositive axons are present in the LDTg and that NTS PPG neurons project to the LDTg, providing anatomical evidence that NTS PPG neurons project to the LDTg and likely release GLP-1. It is worth noting that the CTB injection sites were localized and confined within the LDTg, but did not encompass the entire rostral-caudal, medial-lateral, or dorsal-ventral extent of the LDTg. Thus, the retrograde labeling observed is likely an underestimate of the actual projections. Given these anatomical data combined with our behavioral pharmacology data, we argue that the NTS-toLDTg GLP-1 is physiologically meaningful. Collectively, these data support a potential role of a gut-to-NTS-to-LDTg GLP-1 pathway that mediates energy balance control. These data underscore that the LDTg is a critical site of action for endogenous GLP-1R signaling under physiological conditions.

As FDA-approved GLP-1 mimetics are given systemically, it is important to examine the CNS nuclei that are activated by these peripherally administered GLP-1R agonists. Immunohistochemical data provide further evidence that systemically delivered GLP-1R agonists access the LDTg. Behavioral data provide functional evidence that systemically delivered GLP-1R agonists access the LDTg, as LDTg GLP-1R blockade attenuates the intake suppressive effects of peripherally administered Ex-4. Together, these data highlight the potential preclinical relevance of the GLP-1R LDTg system. Interestingly, in contrast with our previous study showing FLEX localized to and internalized in both neurons and astrocytes within the NTS after IP administration (Reiner et al, 2016), FLEX associates predominately with neurons in the LDTg but only very minimally with LDTg astrocytes. Thus, a cautious speculation of these findings is that GLP-1R may show differential cellular phenotypic expression across the neuraxis. Future investigation should therefore examine the pharmacokinetics and receptorbinding rates for various GLP-1R agonists throughout GLP-1R-expressing nuclei.

Collectively, our data show that LDTg GLP-1R signaling is pharmacologically and physiologically relevant for energy balance control and provide novel evidence of gut-NTSLDTg GLP-1 signaling. Given that the LDTg is anatomically connected to feeding-relevant nuclei in the hindbrain, midbrain, hypothalamus, and other forebrain structures (Cornwall et al, 1990), the current set of findings highlight the importance of investigating the LDTg and its role in integrating information across the neuraxis in order to mediate energy balance. Future studies should also explore how GLP-1 signaling in the LDTg interacts with other energy balance relevant signals to control energy balance, as well as motivated behavior. Importantly, behavioral and anatomical data show that systemically delivered long-acting GLP-1 mimetics can access the LDTg and control food intake, suggesting that the LDTg is of potential preclinical relevance for GLP-1-mediated energy balance control.

\section{FUNDING AND DISCLOSURE}

This research was supported by DK105858 (to DJR), DK103804 (to EGM-B), DK104897 (to SEK), and DK096139 (to MRH). MRH receives research support from Novo Nordisk and MRH and EGM-B receive research support from Zealand Pharma that was not used in support of these studies. The authors declare no conflict of interest.

\section{ACKNOWLEDGMENTS}

We thank Anh Cao, Jack Chen, Alexis Corini, Misgana Ghidewon, Joanna Krawczyk, Nina Juntereal, Rinzin Lhamo, Claudia Liberini, Tyler Ling, Celina Nhan, Chan Nguyen, Tram Pham, Lauren Stein, Evan Shaulson, and Christopher Turner for valuable technical assistance.

\section{REFERENCES}

Alderson HL, Latimer MP, Winn P (2005). Involvement of the laterodorsal tegmental nucleus in the locomotor response to repeated nicotine administration. Neurosci Lett 380: 335-339.

Alhadeff AL, Rupprecht LE, Hayes MR (2012). GLP-1 neurons in the nucleus of the solitary tract project directly to the ventral tegmental area and nucleus accumbens to control for food intake. Endocrinology 153: 647-658.

Andrews PL, Horn CC (2006). Signals for nausea and emesis: Implications for models of upper gastrointestinal diseases. Auton Neurosci 125: 100-115.

Blaha CD, Allen LF, Das S, Inglis WL, Latimer MP, Vincent SR et al (1996). Modulation of dopamine efflux in the nucleus accumbens after cholinergic stimulation of the ventral tegmental area in intact, pedunculopontine tegmental nucleus-lesioned, and laterodorsal tegmental nucleus-lesioned rats. J Neurosci 16: 714-722.

Cornwall J, Cooper JD, Phillipson OT (1990). Afferent and efferent connections of the laterodorsal tegmental nucleus in the rat. Brain Res Bull 25: 271-284.

Dickson SL, Egecioglu E, Landgren S, Skibicka KP, Engel JA, Jerlhag E (2011). The role of the central ghrelin system in reward from food and chemical drugs. Mol Cell Endocrinol 340: 80-87.

Dickson SL, Hrabovszky E, Hansson C, Jerlhag E, Alvarez-Crespo M, Skibicka KP et al (2010). Blockade of central nicotine acetylcholine receptor signaling attenuate ghrelin-induced food intake in rodents. Neuroscience 171: 1180-1186.

DiLeone RJ, Taylor JR, Picciotto MR (2012). The drive to eat: comparisons and distinctions between mechanisms of food reward and drug addiction. Nature Neurosci 15: 1330-1335.

Dobbs LK, Cunningham CL (2014). The role of the laterodorsal tegmental nucleus in methamphetamine conditioned place preference and locomotor activity. Behav Brain Res 265: 198-202.

Dossat AM, Diaz R, Gallo L, Panagos A, Kay K, Williams DL (2013). Nucleus accumbens GLP-1 receptors influence meal size and palatability. Am J Physiol Endocrinol Metab 304: E1314-E1320.

Dossat AM, Lilly N, Kay K, Williams DL (2011). Glucagon-like peptide 1 receptors in nucleus accumbens affect food intake. J Neurosci 31: 14453-14457.

Forster GL, Falcon AJ, Miller AD, Heruc GA, Blaha CD (2002). Effects of laterodorsal tegmentum excitotoxic lesions on 
behavioral and dopamine responses evoked by morphine and d-amphetamine. Neuroscience 114: 817-823.

Grill HJ, Hayes MR (2009). The nucleus tractus solitarius: a portal for visceral afferent signal processing, energy status assessment and integration of their combined effects on food intake. Int $J$ Obes 33(Suppl 1): S11-S15.

Grill HJ, Hayes MR (2012). Hindbrain neurons as an essential hub in the neuroanatomically distributed control of energy balance. Cell Metab 16: 296-309.

Hayes MR, Bradley L, Grill HJ (2009). Endogenous hindbrain glucagon-like peptide-1 receptor activation contributes to the control of food intake by mediating gastric satiation signaling. Endocrinology 150: 2654-2659.

Hayes MR, Kanoski SE, Alhadeff AL, Grill HJ (2011a). Comparative effects of the long-acting GLP-1 receptor ligands, liraglutide and exendin-4, on food intake and body weight suppression in rats. Obesity 19: 1342-1349.

Hayes MR, Leichner TM, Zhao S, Lee GS, Chowansky A, Zimmer D et al (2011b). Intracellular signals mediating the food intakesuppressive effects of hindbrain glucagon-like peptide-1 receptor activation. Cell metabolism 13: 320-330.

Hayes MR, Mietlicki-Baase EG, Kanoski SE, De Jonghe BC (2014). Incretins and amylin: neuroendocrine communication between the gut, pancreas, and brain in control of food intake and blood glucose. Annu Rev Nutr 34: 237-260.

Hayes MR, Skibicka KP, Grill HJ (2008). Caudal brainstem processing is sufficient for behavioral, sympathetic, and parasympathetic responses driven by peripheral and hindbrain glucagon-like-peptide-1 receptor stimulation. Endocrinology 149: 4059-4068.

Hsu TM, Hahn JD, Konanur VR, Lam A, Kanoski SE (2015). Hippocampal GLP-1 receptors influence food intake, meal size, and effort-based responding for food through volume transmission. Neuropsychopharmacology 40: 327-337.

Jerlhag E, Janson AC, Waters S, Engel JA (2012). Concomitant release of ventral tegmental acetylcholine and accumbal dopamine by ghrelin in rats. PLoS ONE 7: e49557.

Kanoski SE, Hayes MR, Skibicka KP (2016). GLP-1 and weight loss: unraveling the diverse neural circuitry. Am J Physiol Regul Integr Comp Physiol 310: R885-R895.

Kanoski SE, Rupprecht LE, Fortin SM, De Jonghe BC, Hayes MR (2012a). The role of nausea in food intake and body weight suppression by peripheral GLP-1 receptor agonists, exendin-4 and liraglutide. Neuropharmacology 62: 1916-1927.

Kanoski SE, Zhao S, Guarnieri DJ, DiLeone RJ, Yan J, De Jonghe BC et al (2012b). Endogenous leptin receptor signaling in the medial nucleus tractus solitarius affects meal size and potentiates intestinal satiation signals. Am J Physiol Endocrinol Metab 303: E496-E503.

Lammel S, Lim BK, Ran C, Huang KW, Betley MJ, Tye KM et al (2012). Input-specific control of reward and aversion in the ventral tegmental area. Nature 491: 212-217.

Laviolette SR, Priebe RP, Yeomans JS (2000). Role of the laterodorsal tegmental nucleus in scopolamine- and amphetamine-induced locomotion and stereotypy. Pharmacol Biochem Behav 65: 163-174.
Lodge DJ, Grace AA (2006). The laterodorsal tegmentum is essential for burst firing of ventral tegmental area dopamine neurons. Proc Natl Acad Sci USA 103: 5167-5172.

Merchenthaler I, Lane M, Shughrue P (1999). Distribution of prepro-glucagon and glucagon-like peptide-1 receptor messenger RNAs in the rat central nervous system. JComp Neurol 403: 261-280.

Mietlicki-Baase EG, Rupprecht LE, Olivos DR, Zimmer DJ, Alter $\mathrm{MD}$, Pierce RC et al (2013). Amylin receptor signaling in the ventral tegmental area is physiologically relevant for the control of food intake. Neuropsychopharmacology 38: 1685-1697.

Mitchell D, Krusemark ML, Hafner D (1977). Pica: a species relevant behavioral assay of motion sickness in the rat. Physiol Behav 18: 125-130.

Narayanan NS, Guarnieri DJ, DiLeone RJ (2010). Metabolic hormones, dopamine circuits, and feeding. Front Neuroendocrinol 31: 104-112.

Omelchenko N, Sesack SR (2005). Laterodorsal tegmental projections to identified cell populations in the rat ventral tegmental area. J Comp Neurol 483: 217-235.

Paxinos G, Watson C (2005). The Rat Brain in Stereotaxic Coordinates5th edn.Elsevier Academic Press: Amsterdam; Boston.

Paxinos G, Watson C (2007). The Rat Brain in Stereotaxic Coordinates6th edn.Academic Press/Elsevier: Amsterdam; Boston.

Reiner DJ, Mietlicki-Baase EG, McGrath LE, Zimmer DJ, Bence KK, Sousa GL et al (2016). Astrocytes regulate GLP-1 receptormediated effects on energy balance. J Neurosci 36: 3531-3540.

Reiner DJ, Mietlicki-Baase EG, Olivos DR, McGrath LE, Zimmer DJ, Koch-Laskowski K et al (2017). Amylin acts in the lateral dorsal tegmental nucleus to regulate energy balance through gamma-aminobutyric acid signaling. Biol Psychiatry. (e-pub ahead of print).

Schmidt HD, Famous KR, Pierce RC (2009). The limbic circuitry underlying cocaine seeking encompasses the PPTg/LDT. Eur J Neurosci 30: 1358-1369.

Scott KA, Moran TH (2007). The GLP-1 agonist exendin-4 reduces food intake in nonhuman primates through changes in meal size. Am J Physiol Regul Integr Comp Physiol 293: R983-R987.

Steidl S, Cardiff KM, Wise RA (2015). Increased latencies to initiate cocaine self-administration following laterodorsal tegmental nucleus lesions. Behav Brain Res 287: 82-88.

Takeda N, Hasegawa S, Morita M, Matsunaga T (1993). Pica in rats is analogous to emesis: an animal model in emesis research. Pharmacol Biochem Behav 45: 817-821.

Terrill SJ, Jackson CM, Greene HE, Lilly N, Maske CB, Vallejo S et al (2016). Role of lateral septum glucagon-like peptide 1 receptors in food intake. Am J Physiol Regul Integr Comp Physiol 311: R124-R132.

Vrang N, Phifer CB, Corkern MM, Berthoud HR (2003). Gastric distension induces c-Fos in medullary GLP-1/2-containing neurons. Am J Physiol Regul Integr Comp Physiol 285: R470-R478.

Yamamoto K, Matsunaga S, Matsui M, Takeda N, Yamatodani A (2002). Pica in mice as a new model for the study of emesis. Methods Find Exp Clin Pharmacol 24: 135-138.

Supplementary Information accompanies the paper on the Neuropsychopharmacology website (http://www.nature.com/npp) 